\title{
Differential regulation of interleukin-8 and human beta-defensin 2 in Pseudomonas aeruginosa-infected intestinal epithelial cells
}

\author{
Fu-Chen Huang
}

\begin{abstract}
Background: The human opportunistic pathogen, Pseudomonas aeruginosa ( $P$. aeruginosa) carries the highest case fatality rate of all gram-negative infections. Unfortunately, antimicrobial therapy has not been demonstrated to improve clinical outcome and the emergence of multidrug resistant $P$. aeruginosa has become a major concern in the hospital setting. Fever and diarrhea are the two most common initial symptoms in $P$. aeruginosa sepsis in previously healthy infants and children. This implies that intestinal epithelial cells in first contact with the pathogen may play an important role in innate immunity to $P$. aeruginosa infection. Human beta-defensins-2 (hBD-2) and interleukin-8 (IL-8) are crucial for host defense at mucosa but IL-8 may give rise to characteristic pathology of colitis.

Results: Pseudomonas aeruginosa strain PAO1 was used to infect SW480, an intestinal epithelial cell. IL-8 and hBD-2 mRNA expression and protein secretion were then assessed in SW480 cells using RT-PCR and enzyme-linked immunosorbent assay (ELISA), respectively. Intracellular signaling pathways and nucleotide-binding oligomerization domain (NOD) 1 protein expression were analyzed by Western blot in SW480 cells in the presence or absence of inhibitors or transfected with siRNA.

We demonstrate that prolonged infection by $P$. aeruginosa results in suppression of IL-8 but enhancement of hBD-2, either protein secretion and mRNA expression, in SW480 cells. Inhibitors of ERK suppressed but inhibitor of PI3K enhanced P. aeruginosa-induced IL-8 mRNA expression in SW480 cells while both signaling had no effect on P. aeruginosa-induced hBD-2 expression in SW480 cells. On the other hand, NOD 1 was illustrated to get involved in P. aeruginosa-induced hBD-2 mRNA expression and protein production in SW480 cells.

Conclusions: The P. aeruginosa-induced antimicrobial peptide in IECS continuously protect the host against prolonged infection, while modulation of proinflammatory responses prevents the host from the detrimental effects of overwhelming inflammation. Thus, $P$. aeruginosa-induced innate immunity in IECs represents a host protective mechanism, which may provide new insight into the pathogenesis of inflammatory bowel diseases.
\end{abstract}

Keywords: Pseudomonas aeruginosa, Interleukin 8, Human beta-defensin 2, Intestinal epithelia

\section{Background}

The human opportunistic pathogen, Pseudomonas aeruginosa ( $P$. aeruginosa), is a major cause of infectionrelated mortality among critically ill patients, and carries the highest case fatality rate of all gram-negative infections [1]. Although rare, P. aeruginosa bacteremia is often rapidly progressive and can occur with a high mortality rate in previously healthy patients [2,3], even receiving

Correspondence: huang817@cgmh.org.tw

Department of Pediatrics, Kaohsiung Chang Gung Memorial Hospital and Chang Gung University College of Medicine, 123, Ta-pei Road, Niao-sung District, Kaohsiung, Taiwan

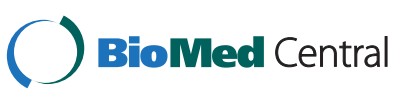

appropriate antimicrobial treatment. Moreover, in a pediatric study of Pseudomonas bacteremia, antimicrobial susceptibility was not identified as a prognostic factor [4]. $P$. aeruginosa is not only difficult to treat but also exhibits remarkable ability to acquire resistance to these agents [5]. Overall, resistance rates are on the increase. Multidrug resistance is frequent, and clinical isolates resistant to virtually all anti-pseudomonal agents are increasingly being reported. Therefore, effective immunotherapy may be a useful alternative therapy administered either alone or in combination with antibiotic chemotherapy. 
In a study of community-acquired sepsis associated with $P$. aeruginosa in previously healthy infants and children [2], fever and diarrhea were the two most common initial symptoms. $P$. aeruginosa was also isolated in $43 \%$ of fecal specimens. This implied that intestinal epithelial cell first contacting the pathogen may play an important role on innate immunity to $P$. aeruginosa infection. In addition to serving as a protective barrier, the epithelium plays an active role in the intestinal immune response through its secretion of inflammatory cytokines, chemokines, and antimicrobial peptides [6,7]. Antimicrobial peptides, such as human $\beta$-defensins-2 (hBD-2), are crucial for host defense at mucosal surfaces while chemokines, such as interleukin-8 (IL-8), recruit neutrophils from the circulation into the subepithelial region to defend against the invasion of bacteria, but give rise to characteristic pathology of colitis [8].

Two main families of pattern-recognition receptors involved in innate immune detection have been discovered in human beings. Toll-like receptors (TLRs) are transmembranous molecules [9] and cytosolic Nucleotide-binding oligomerization domains (NODs) [10] are seen as the intracellular counterpart of the TLRs. Both play essential roles in the clearance of $P$. aeruginosa [11]. Shedding of flagellin, recognized by TLR5, from $P$. aeruginosa provokes hBD-2 and IL-8 response in human keratinocytes [12]. Intestinal epithelial cells (IECs) are generally hyporesponsive to extracellular bacterial products, especially TLR2 and TLR4 ligands [13]. The unresponsiveness of IECs to TLR signals sets the stage for the function of NOD proteins as important sensors for the detection of bacteria invading the epithelium [14]. Autophagy plays an essential role in the clearance of $P$. aeruginosa by alveolar macrophages. Two groups of investigators $[15,16]$ have demonstrated that NOD1 and NOD2 are critical for the autophagic response to invasive bacteria because they recruit ATG16L1 to bacterial entry sites at the plasma membrane. Several studies have implicated NOD1-dependent NF- $\mathrm{kB}$ activation in the induction of $\beta$-defensins and chemokines expression in response to $H$. pylori [17] and S. flexneri infection [18]. Moreover, a recent study showed that NOD2 is essential in the enhancement of IL- 8 induced by S. aureus through activation of c-jun NH2-terminal kinase (JNK) pathway and upregulation of COX2 [19]. The cooperation of TLR5 and NOD2 in IECs regulates inflammatory response to Salmonella infection [20].

Therefore, we aim to investigate the intestinal epithelial IL-8 and hBD-2 expression in P. aeruginosa-infected IECs and the downstream signaling pathways of TLRs or NODs involved in the effects. Up to now, the innate immunity of IECs to $P$. aeruginosa infection has been completely unknown. We have studied the inflammatory responses in $P$. aeruginosa-infected IECs, and for the first time reveal the differential regulation of $P$. aeruginosa-induced IL-8 and hBD-2 in IECs via PI3K/Akt signal and NOD1 protein respectively. This observation could provide useful information for further understanding of the innate immunity in mucosal $P$. aeruginosa infection.

\section{Methods}

\section{Cell culture and infection}

SW480 and Caco-2 cells (ATCC, Rockville, MD), transformed human colonic epithelial cell lines, were grown in Dulbecco modified Eagle medium (DMEM) supplemented with $10 \%$ heat-inactivated fetal calf serum, 100 units $/ \mathrm{ml}$ penicillin, $100 \mu \mathrm{g} / \mathrm{ml}$ streptomycin sulfate, and $20 \mathrm{mM}$ HEPES (Sigma) in a $5 \% \mathrm{CO}_{2}$ atmosphere at $37^{\circ} \mathrm{C}$. Passage 10-30 will be used for all experiments. For stimulation experiments, cells were seeded in 12-well tissue culture plates ( $4 \mathrm{~cm}^{2} /$ well; BD Biosciences) and used at $60-80 \%$ confluence.

\section{Reagents}

The PI3K inhibitors, wortmannin and LY294002, and the mitogen-activated protein (MAP) kinase ERK inhibitor, PD98059 were obtained from Calbiochem (San Diego, CA) and stock solutions made in dimethylsulfoxide (DMSO). Standard laboratory reagents were from Sigma (St. Louis, MO, USA) or Fisher Scientific (Pittsburgh, PA, USA).

\section{Bacterial strain}

P. aeruginosa strain PAO1 is a well-characterized laboratory strain. It is grown in tryptic soy broth (Difco Laboratories, Detroit, MI) supplemented with $10 \mu \mathrm{g} / \mathrm{ml}$ kanamycin for $6 \mathrm{~h}$ until the optical density at $600 \mathrm{~nm}$ is 0.5 or the concentration is about $1 \times 10^{8} \mathrm{CFU} / \mathrm{ml}$. The bacteria is collected by centrifugation at 3,000 rpm for $10 \mathrm{~min}$, washed twice by resuspension in sterile phosphate-buffered saline (PBS) (GIBCO, Grand Island, NY), and finally suspended at the desired dilution in PBS. Cultured cells were infected at a multiplicity of infection (MOI) of 10 for indicated times.

\section{Cytokine assays \\ IL-8 ELISA}

After treatment, the medium was aspirated at the end of the infection period, from control or infected cells, cleared of any $P$. aeruginosa or cellular debris by centrifugation $\left(5 \mathrm{~min}, 1,000 \times g\right.$ ), then stored at $-20^{\circ} \mathrm{C}$ until use. The supernatant medium was collected and IL-8 concentrations determined by enzyme-linked immunosorbent assay (ELISA) as described in detail earlier [20,21].

\section{hBD-2 ELISA}

After treatment or infection, the supernatant medium of the cultured cells was collected and hBD-2 concentrations determined by enzyme-linked immunosorbent assay (ELISA) as manufacturer's instructions with some modification. Briefly, 96-well immunoplates were coated 
with goat anti-hBD-2 antibody (PeproTech, Rocky Hill, NJ). After blocking and washing, $100 \mu \mathrm{l} /$ well of cell culture supernatants and serial dilutions of standard hBD-2 in cell culture medium were incubated for $30 \mathrm{~min}$ at room temperature. Subsequently, wells were incubated with biotinylated goat anti-hBD-2 antibody (Cell Concepts), filled with $50 \mu \mathrm{l} /$ well of Streptavidin-POD (Roche Diagnostics) and developed by ABTS (Roche Diagnostics). Absorbance is measured at $405 \mathrm{~nm}$ with a multichannel photometer (Sunrise; Tecan, Crailsheim, Germany). Because of variations in baseline hBD-2 production, the results are expressed as "fold increase", representing the normalized hBD-2 produced by infected monolayers divided by the normalized hBD-2 produced by control, uninfected cells.

\section{Protein extraction}

Cytosolic and membranous extracts from untreated and treated SW480 cells were prepared as manufacturer's instructions with slight modifications [21,22]. Protein concentrations in cell fractions were determined using a Bio-Rad assay kit.

\section{Western blotting}

Equal amounts of total protein were separated by SDS-PAGE and then transferred to nitrocellulose membranes by semi-dry blotting as previously described [23,24]. After blocking the membranes with 5\% non-fat dry milk, they were probed with antibodies to either phosphorylated p38, JNK, Akt (Cell Signaling, Beverly, MA), phosphorylated ERK (Santa Cruz Biotechnology, Santa Cruz, CA), phosphorylated IкB (New England BioLabs, Beverly, MA), anti-MyD88, ATG16L1, Beclin-1, Atg5, rabbit anti-LC3 (Cell Signaling, Beverly, MA), or anti-NOD1 and NOD2 (Cayman Chemical, Ann Arbor, MI), and then developed with horseradish peroxidase-conjugated second antibodies (Zymed Laboratories, San Francisco, CA) and enhanced chemiluminescence (Pierce Chemical Co., Rockford, IL). Appropriate exposures to X-ray film were made, and the filters then stripped and re-probed with antibodies to GAPDH (Santa Cruz Biotechnology, Santa Cruz, CA) as appropriate.

\section{Real-time PCR for mRNA assay}

Total RNA was prepared from control or infected cells with the Trizol reagent (Invitrogen Corporation, Carlsbad, CA), following the manufacturer's directions. The RNA was reverse-transcribed with random hexamers using the GeneAmp kit (Roche, Nutley, NJ) as described in detail earlier $[20,21,23,24]$. Real-time reverse transcription-PCR analyses were performed in a fluorescence temperature cycler (LightCycler; Roche Diagnostics) as described previously $[20,21]$. The following primers were used: IL-8, 5'AAACCACCGGAAGGAACCAT-3' (forward primer) and 5'-GCCAGCTTGGAAGTCATGT-3' (reverse primer); hBD-2, 5' -ATCAGCCATGAGGGTCTTGT-3' (forward primer) and 5' -GAGACCACAGGTGCCAATTT-3' (reverse primer); or glyceraldehyde-3-phosphate dehydrogenase, 5'-CCAGCCGAGCCACATCGCTC-3' (forward primer) and 5'-ATGAGCCCCAGCCTTCTCCAT-3'. Standard curves were obtained for each primer set with serial dilutions of cDNA. All quantifications were normalized to the housekeeping gene glyceraldehyde-3phosphate dehydrogenase. Relative expression was given as a ratio between target gene expression and glyceraldehyde3-phosphate dehydrogenase expression.

\section{Small-interfering RNA (siRNA) transfection}

All transient transfections were carried out in triplicate using NeoFX reagent (Ambion, Austin, TX) to final concentration of $20 \mathrm{nM}$ following the manufacturer's instructions. The siRNAs used were as follows: NOD1 siRNA and siRNA for the negative control (Invitrogen Corporation, Carlsbad, CA). All siRNA were tested and verified as reducing expression by $>80 \%$ protein reduction in SW480 cells by immunoblot analysis or reducing expression of $>50 \%$ of mRNA by real-time $\mathrm{PCR}$ when appropriate Ab was not available, as in our previous work [20]. For the SW480 cells, 20nM of each siRNA was transfected $48-96 \mathrm{~h}$ before $P$. aeruginosa infection.

\section{Statistical analysis}

All above experiments were carried out at least three times with similar results. Statistical significance was determined using the Student's $t$-test.

\section{Results}

Prolonged infection by $P$. aeruginosa resulted in suppression of IL-8 but enhancement of hBD-2 protein secretion

The cultured cells were uninfected (control) or infected by $P$. aeruginosa for indicated times. Supernatant of cultured cells was analyzed by ELISA for IL-8 and hBD-2 secretion. As shown in Figure 1, the production of IL-8 and hBD-2 followed two distinct time-courses. First, IL- 8 showed a peak after 5 hours and a decline thereafter. A second, distinct time-course was observed for hBD-2, which remained elevated 7 hours after $P$. aeruginosa infection.

\section{Prolonged infection by $P$. aeruginosa results in enhancement of IL-8 and hBD-2 mRNA expression in SW480 cells}

We proceeded to examine the effect of $P$. aeruginosa infection on IL-8 and hBD-2 mRNA levels. SW480 cells were either uninfected or infected by $P$. aeruginosa. Total RNA was prepared, reverse transcribed with random hexamers, and analyzed by real-time quantitative PCR. As shown in Figure 2, P. aeruginosa infection induced IL-8 


\section{$\square \mathrm{IL}-8 \square \mathrm{hBD}-2$}

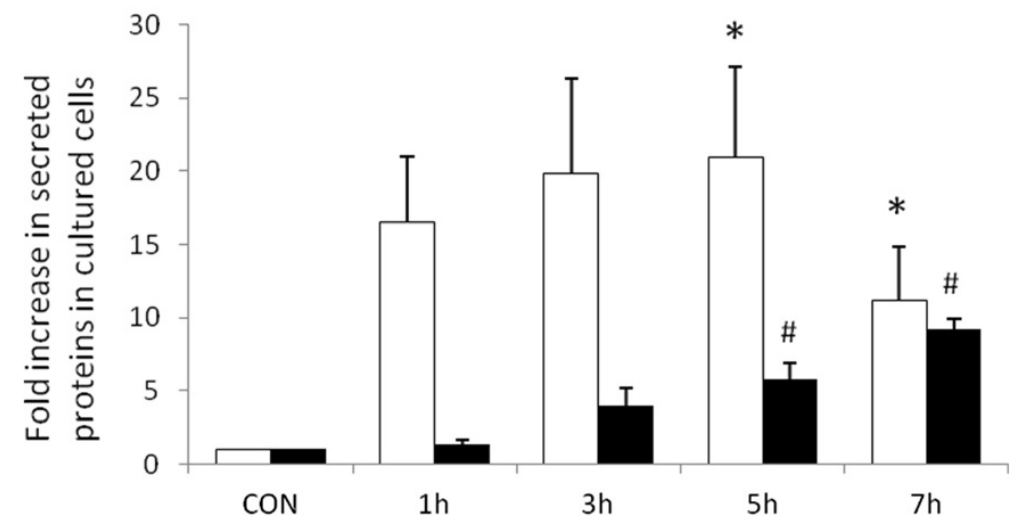

Figure 1 The effect of prolonged infection by P. aeruginosa on IL-8 and hBD-2 proteins secretion in SW480 cells. SW480 cells were left uninfected (CON) or infected with the wild-type P. aeruginosa strain PAO1 for the times indicated. Supernatant was analyzed by ELISA 6 hours later for IL-8. The amount of IL-8 produced is shown as the fold increase over uninfected control cells. Results are represented as means \pm S.E.M. for at least three determinations from independent experiments. $\left({ }^{*} p<0.05 ; \# p<0.05\right)$.

mRNA and hBD-2 mRNA expression (normalized to GAPDH) after one-hour infection in SW480 cells. Both IL-8 mRNA and hBD-2 mRNA in SW480 cells continued to increase after prolonged infection.

\section{The involvement of ERK PI3K/Akt, NF-KB signaling} pathway and NOD1 protein in SW480 cells after

\section{P. aeruginosa infection}

To further evaluate which signaling pathway is involved in $P$. aeruginosa-induced IL- 8 and $\mathrm{hBD}-2$ regulation, we investigated the downstream signal pathways of TLRs and autophagy proteins. SW480 cells were uninfected or infected with PAO1. Activation of the ERK JNK, p38, Akt and $I_{k} B$, were analyzed in whole cell protein by Western blot. Western blot data showed that protein levels of p-ERK were significantly upregulated at 5 and 15 minutes infection, reached peak at $45 \mathrm{~min}$, and significantly decreased at $120 \mathrm{~min}$ when p-Akt and p-IкB were significantly upregulated (Figure 3 ), suggesting the involvement of ERK, Akt and IKB in the downstream signaling of $P$. aeruginosa infection. However, the activation of JNK or p38 had no significant upregulation from 5 to $120 \mathrm{~min}$. Western blot analysis was also applied to evaluate autophagy proteins expression in $P$. aeruginosa-infected

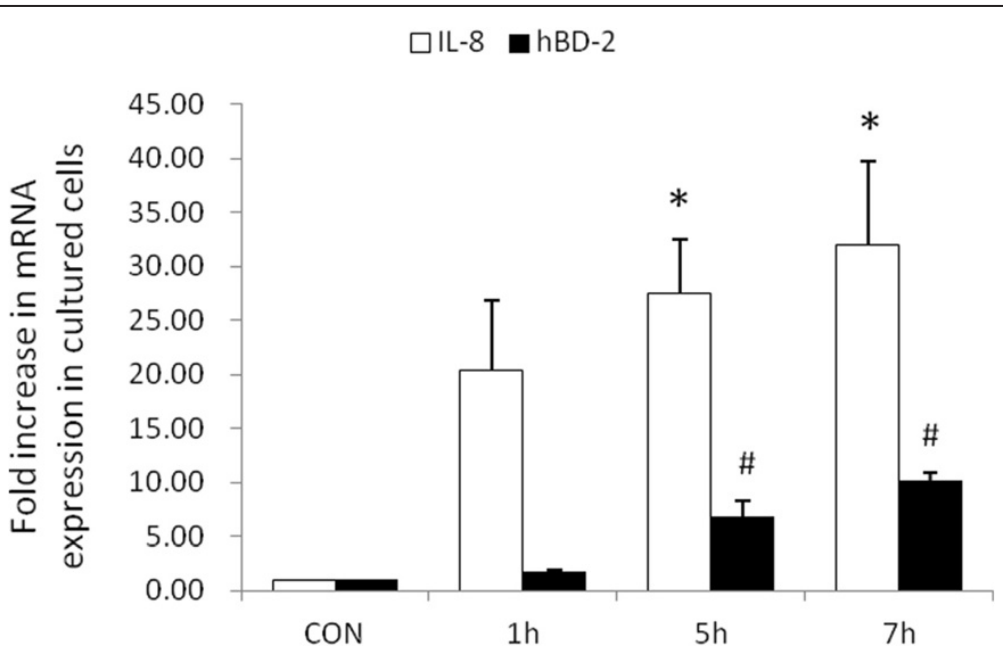

Figure 2 The effect of prolonged $P$. aeruginosa infection on IL-8 and hBD-2 mRNA expression in SW480 cells. SW480 cells were left uninfected $(\mathrm{CON})$ infected with the wild-type $P$. aeruginosa strain PAO1 for one to seven hours. Total RNA was prepared after infection and analyzed by real-time quantitative PCR to estimate amounts of IL-8 and hBD-2 transcript. The amount of IL-8 and hBD-2 mRNA produced, normalized to the corresponding amount of GAPDH transcript, is shown as the fold increase over uninfected control cells. Results are represented as means \pm S.E.M. for at least three determinations from independent experiments. $\left({ }^{*} p<0.05\right.$; \# $\left.p<0.05\right)$. 


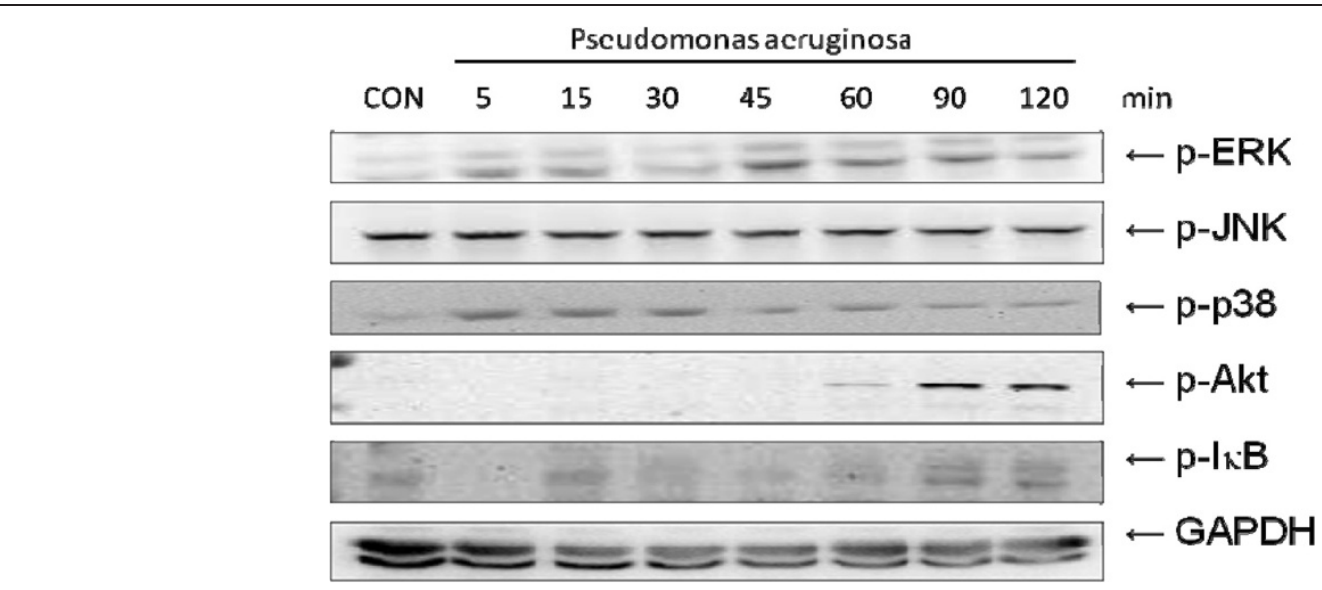

Figure 3 The proteins expression of intracellular signaling pathways in P. aeruginosa-infected SW480 cells. SW480 cells were left uninfected (CON), or infected with wild-type P. aeruginosa strain PAO1 for the times indicated. Activation of the ERK, JNK, p38, Akt and IKB were analyzed in whole cell protein by immunoblotting with antibodies to phosphorylated (p) ERK, JNK, p38, Akt and IkB. The results shown are representative of three separate experiments. GAPDH worked as a normalization of cytosolic protein.

SW480 cells. It was demonstrated that the band density of NOD1 continuously increased after prolonged infection by P. aeruginosa, while other autophagy proteins (e.g. NOD2, Beclin1 or Atg5) had the same band density even after prolonged infection (Figure 4). It suggested the involvement of NOD1 in enhanced hBD-2 expression in SW480 cells after prolonged $P$. aeruginosa infection.

The involvement of ERK and PI3K/Akt signaling pathway in negative regulation of IL-8 after prolonged $P$. aeruginosa infection

Pretreatment with ERK and PI3K inhibitors (PD98059 for ERK and LY294002 for PI3K) (Figure 5A) suppressed and upregulated $P$. aeruginosa-induced IL- 8 production in SW480 cells respectively. These data indicated that
ERK-mediated IL-8 production was suppressed via activation of the PI3K/Akt signaling pathway. We proceeded to examine the involvement of ERK and Akt in the $P$. aeruginosa-induced IL-8 mRNA levels. SW480 cells were either uninfected or infected by $P$. aeruginosa for 7 hours in the absence or presence of PD98059 or LY294002. Total RNA was prepared, reverse transcribed with random hexamers, and analyzed by real-time quantitative PCR. As shown in Figure 5B, P. aeruginosa infection induced IL-8 mRNA expression (normalized to GAPDH) in SW480 cells. Both inhibitors had no significant effect on $P$. aeruginosainduced IL-8 mRNA expression in SW480 cells, suggesting that ERK and PI3K/Akt signal pathways were involved in $P$. aeruginosa-induced IL-8 immune response by posttranscriptional or post-translational mechanisms.

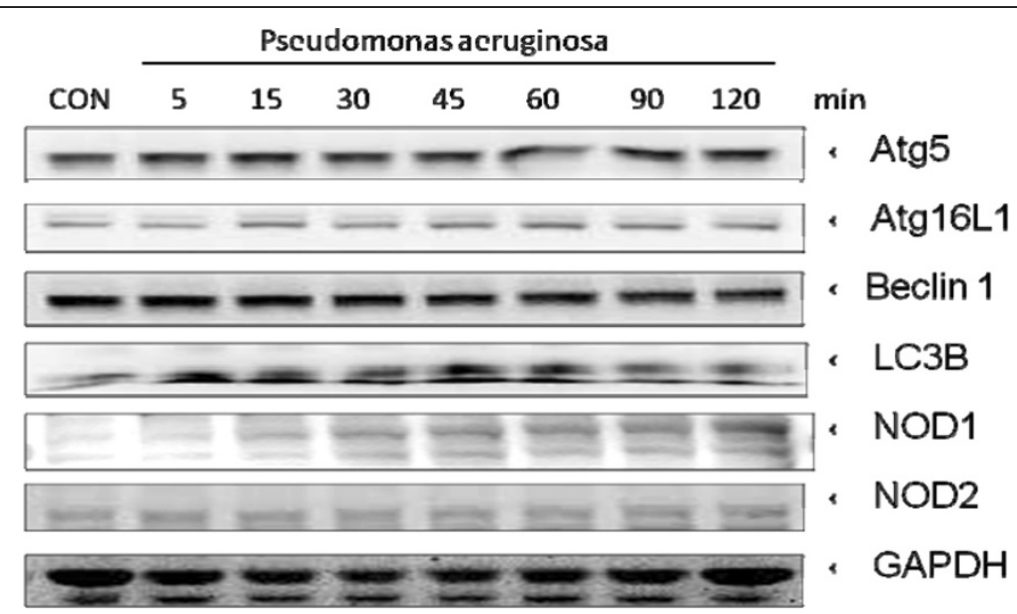

Figure 4 The proteins expression of autophagy in P. aeruginosa-infected SW480 cells. SW480 cells were uninfected (CON) or infected by $P$. aeruginosa for indicated times. The Western blots illustrate the expression of Agt5, Atg16L1, Beclin 1, LC3B, NOD1 and NOD2 proteins in cytosolic extracts of SW480 cells infected by P. aeruginosa at indicated times. The results shown are representative of three separate experiments. GAPDH worked as a normalization of cytosolic protein. 


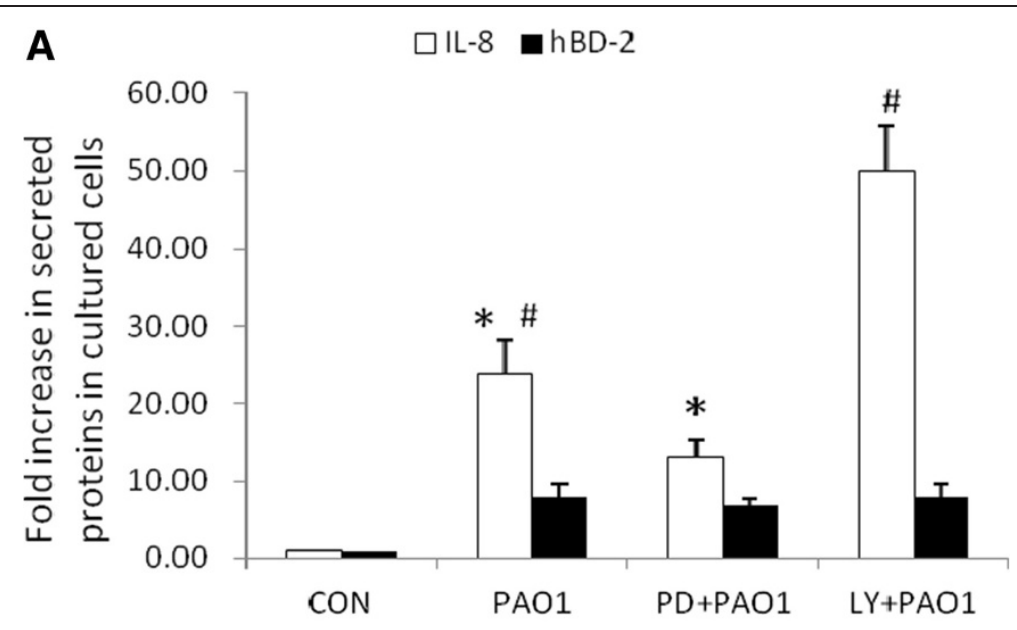

B $\square \mathrm{IL}-8 \mathrm{hBD}-2$

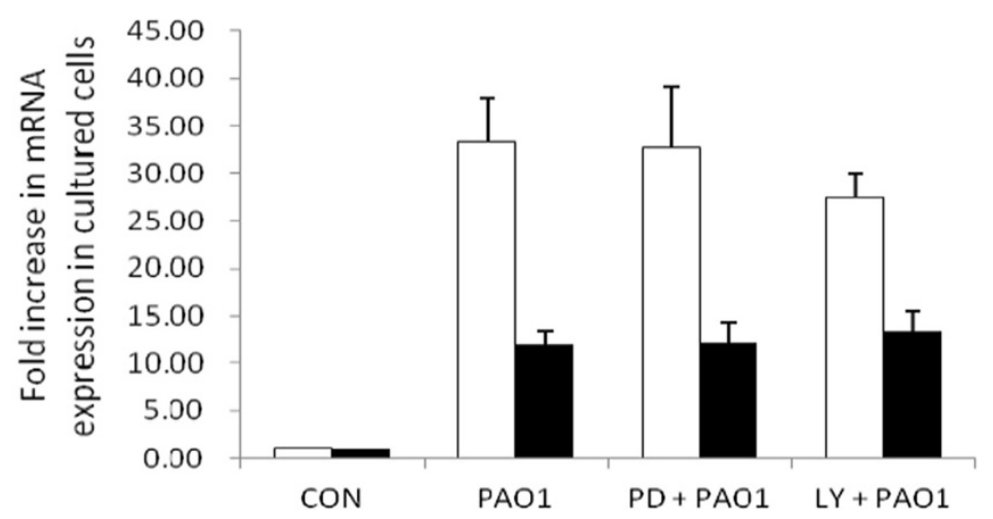

Figure 5 The involvement of ERK and PI3K/Akt signal pathways in P. aeruginosa-induced IL-8 in SW480 cells. (A) Effect of ERK and PI3K inhibition on P. aeruginosa-induced IL-8 and hBD-2 secretion. SW480 cells were left untreated, or treated with $25 \mu \mathrm{M}$ PD98059 (PD) and $50 \mu \mathrm{M}$ LY294002 (LY) for one hour. They were then infected with the wild-type $P$. aeruginosa strain PAO1 for 7 hours. Supernatant was analyzed by ELISA for IL-8 and hBD-2. The amount of IL-8 and hBD-2 produced is shown as the fold increase over uninfected control (CON) cells. (B) Effect of ERK and PI3K inhibition on P. aeruginosa-induced IL-8 and hBD-2 mRNA. SW480 cells were left untreated, or treated with 25 HM PD98059 (PD) and $50 \mu \mathrm{M}$ LY294002 (LY). They were then infected with the wild-type $P$. aeruginosa strain PAO1 for 7 hours. Total RNA was prepared and analyzed by real-time quantitative PCR to estimate amounts of IL-8 and hBD-2 transcript. The amount of IL-8 and hBD-2 mRNA produced, normalized to the corresponding amount of GAPDH transcript, is shown as the fold increase over uninfected control (CON) cells. Results are represented as means \pm S.E.M. for at least three determinations from independent experiments. $\left(^{*} p<0.05\right.$; \# $\left.p<0.005\right)$.

Pretreatment with ERK and PI3K inhibitors (PD98059 for ERK and LY294002 for PI3K) had no significant effect on $P$. aeruginosa-induced hBD-2 production (Figure 5A) or mRNA expression (Figure 5B) in SW480 cells.

The involvement of NOD1 in positive regulation of hBD-2 after prolonged $P$. aeruginosa infection

Based on our observation that the expression of NOD1 protein was upregulated after prolonged $P$. aeruginosa infection, we investigated if NOD1 was involved in the $P$. aeruginosa-induced hBD-2 expression. We adapted a siRNA knockdown approach for NOD1. Knockdown of NOD1 was confirmed by Western blot with specific siRNA in SW480 cells up to 48 hrs (Figure 6A). siRNA-transfected
SW480 cells were uninfected or infected by $P$. aeruginosa PAO1 for 7 hours. Following knockdown of NOD1, we detected the $P$. aeruginosa-induced hBD-2 production (Figure 6B) and mRNA expression (Figure 6C) in SW480 cells was diminished in NOD1-silenced cells, but not in control siRNA-silenced cells. It suggested NOD1 was involved in the enhanced hBD-2 expression in SW480 cells by prolonged $P$. aeruginosa infection.

It is a general phenomenon in Caco-2 intestinal epithelial cell lines

To determine whether the above finding was a general phenomenon among different intestinal epithelial cell lines, the same experiments were undertaken in Caco-2 


\section{A P. aeruginosa}

\section{CON PAO1 SIRNA SINOD1}

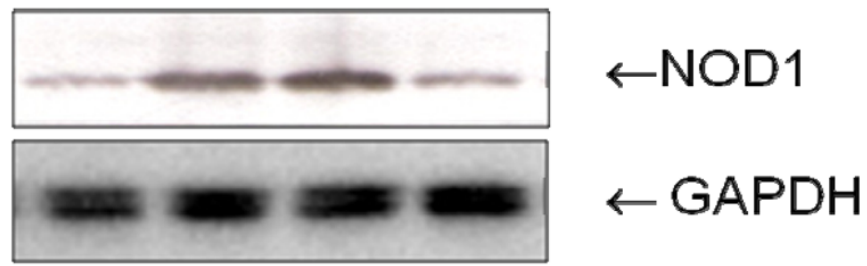

\section{B}

口IL-8 $\mathbf{m} H B D-2$

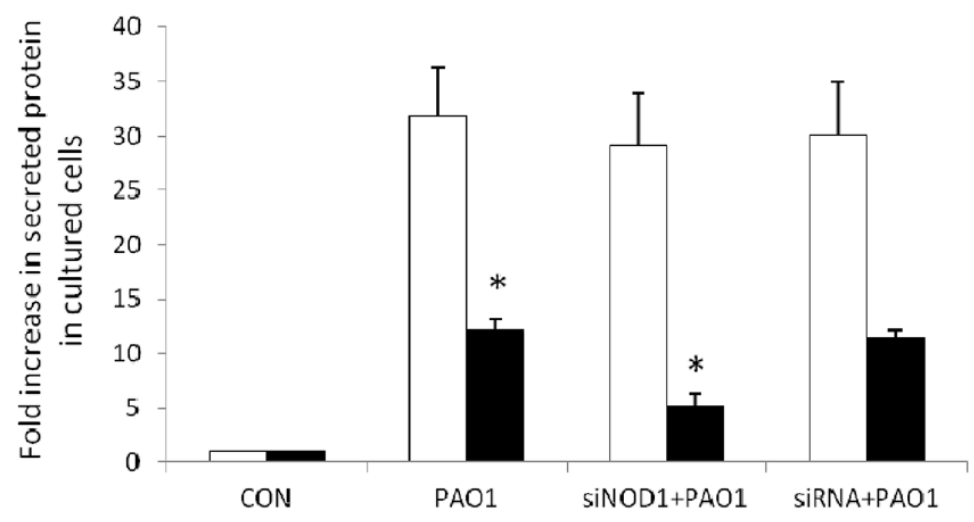

C $\square \mathrm{IL}-8 \quad \mathbf{H B D}-2$

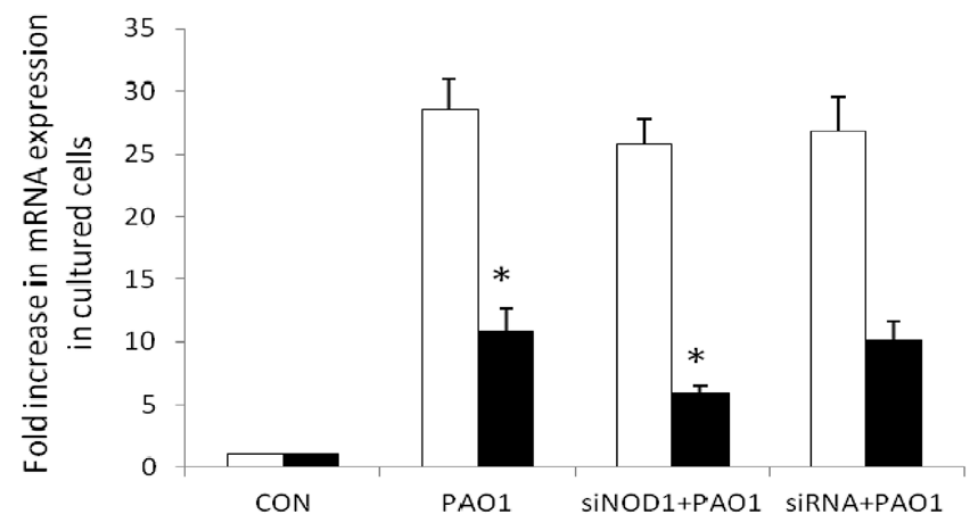

Figure 6 The involvement of NOD1 in P. aeruginosa-induced hBD-2 in SW480 cells. SW480 cells were transfected with control siRNA and NOD1 siRNA (siRNA = non-target control siRNA; siNOD1 = siRNA to NOD1) for 48 hours. The transfected cells were left uninfected or infected by $P$. aeruginosa PAO1 for 7 hours. (A) Western blots probed with antibodies against NOD1 and GAPDH confirm knockdown of NOD1. (B) Supernatant was analyzed by ELISA for secreted IL-8 and hBD-2 protein in SW480 cells. The amount of IL-8 or hBD-2 produced is shown as the fold increase over control cells. (C) After infection, total RNA was extracted from the cells and analyzed byReal-time PCR, as described in Materials and Methods. Results are represented as means \pm S.E.M. for at least three determinations from independent experiments. An asterisk indicates a significant difference $(p<0.05)$.

cells. Caco-2 cells were untreated or pretreated by inhibitors and infected with wild-type $P$. aeruginosa PAO1, using the same experiments as above. The supernatants were analyzed for proteins secretion in Caco-2 cells after treatment of inhibitors or siRNA. The proteins extracted from the cells were analyzed for signaling pathways involved in the regulation. Similar to SW480 cells, we have seen the same results in Caco-2 cells in response to infection (Additional file 1: Figure S1, Additional file 2: Figure S2, Additional file 3: Figure S3 and Additional file 4: Figure S4). 


\section{Discussion}

While the expression of antimicrobial molecules in epithelial cells has been shown as a key to control infection, modulation of proinflammatory responses prevents the cells from the detrimental effects of overwhelming inflammation. In several models of bacterial infection in mice, maximum KC (murine IL-8) expression that recruits and activates neutrophils occurs in the first hours, resulting in enhanced clearance of bacteria after Pseudomonas challenge [25]. NF-kappa B is a central regulator of the intestinal epithelial cell innate immune response induced by infection with enteroinvasive bacteria [26]. Phosphorylation of I $\mathrm{kB}$ (Figure 3), inhibitor of NF- $\mathrm{BB}$, is targeted for proteasome-dependent degradation, leading to translocation of NF- $\kappa B$ to the nucleus and transactivation of NF- $\kappa B$ target genes, giving rise to IL- 8 and hBD-2 mRNA expression (Figure 2). However, prolonged production of IL-8 will cause tissue damage. The massive influx of neutrophils into Pseudomonas-infected sites is stimulated by an excessive production and release of inflammatory mediators, the most important of which include prolonged and sustained expression of IL-8 [27-29]. The accumulation of neutrophils, a hallmark of inflammation, in turn contributes to tissue destruction [30], particularly at the early stage of $P$. aeruginosa colonization. Consequently, translocation of bacteria and absorption of endotoxins may have profound systemic effects and may result in bacteremia, as well as endotoxemia. Likewise, intensive mucosal injury by massive influx of neutrophils may result in translocation of $P$ aeruginosa into blood stream and subsequent sepsis. This can also explain why fever and diarrhea are the two most common initial symptoms in $P$. aeruginosa sepsis in previously healthy infants and children [2].

Substantial evidence has shown that PI3K/Akt activation promotes the internalization of PA strains PAO1 and PAK by epithelial cells [31], and suppresses a proinflammatory response through negatively regulating TLR signaling [32]. Based on our observation that PI3K/Akt pathway plays an anti-inflammatory role, decreasing IL-8 production on Salmonella infection in IECs via interactions with ERK kinase [24], we demonstrated that inhibition of PI3K upregulated but inhibition of ERK suppressed $P$. aeruginosa-induced IL-8 production in IECs after prolonged infection (Figure 5). It suggested that the antiinflammatory PI3K/Akt signal pathway was activated at a late stage to suppress the detrimental effect of IL- 8 overexpression after prolonged $P$. aeruginosa infection (Figure 1). In contrast, PI3K/Akt has no significant effect on hBD-2 expression (Figure 5), which is needed to defend the cells against Pseudomonas infection continuously. PI3K/Akt signaling pathway promotes host resistance against $P$. aeruginosa infection by suppressing corneal inflammation and perforation in mice [33]. Inhibition of PI3K resulted in worsened disease after $P$ aeruginosa corneal infection. This observation is consistent with some studies showing that intraperitoneal injection with wortmannin increased serum cytokines levels and led to susceptibility to sepsis [34]. The pharmaceuticals to block IL-8 or enhance PI3K/Akt signaling pathway may be a promising immunotherapy for antibiotics-resistant $P$. aeruginosa, prevention of $P$. aeruginosa sepsis and lessen usage of antibiotics.

Abnormalities in the handling of intracellular bacteria may allow persistent multiplication of bacteria and trigger chronic uncontrolled intestinal inflammation [35]. A significant increase in the adaptive immunologic response to Crohn's disease (CD)-associated microbial antigens, such as Pseudomonas fluorescens-related protein (I2) and Escherichia coli outer membrane porin C (anti-OmpC), is due to the presence of a defective innate immune gene (NOD2). HBD-2 is an antimicrobial peptide implicated in the pathogenesis of inflammatory bowel disease (IBD) [36]. Children with $C D$ showed a lower expression of hBD-2 in the inflamed terminal ileum and ascending colon [37]. It suggests that NOD2 mutants result in dysregulation of hBD-2 in mucosa intruded by some Pseudomonas species predisposed to IBD. An inappropriate immune response to commensal Pseudomonas species is involved in colonic CD etiology [38]. NOD1 or NOD2 prevalence in colon or ileum could also be due to the predominance of different intracellular organisms or enteroinvasive bacteria for which they are receptors. Variants of NOD1 and NOD2 genes display opposite associations with risk of CD [39]. NOD1 is not involved in IBD [40] while NOD2 mutants are susceptible to IBD. It is compatible with the finding that $P$. aeruginosa was only identified in the gut of non-IBD patients [38] because the innate immunity to $P$. aeruginosa is NOD1-dependent (Figures 4 and 6) but not NOD2 (data not shown). NOD1 plays an important role in the initial recognition of pathogenic bacteria at epithelial surfaces, such as the gut, where innate immune responses to commensal bacteria must be avoided [14]. Likewise it was demonstrated that NOD1 but not NOD2, seems to play a role in chronic infection of airway by $P$. aeruginosa in cystic fibrosis patients [41]. Therefore, it is hypothesized that the loss of commensal $P$. aeruginosa in $\mathrm{CD}$ patients may result in dysbiosis between Pseudomonas species and other gut microbiota, which is directly involved in CD manifestation.

There were many signal pathways reported to be involved in the regulation of hBD-2 in a variety of cultured cells infected by $P$. aeruginosa $[33,42,43]$, including NF- $\mathrm{KB}$, AP-1, PI3K/Akt, p38MAPK, ERK, and JNK. However this is the first time to demonstrate the involvement of NOD1 in $P$. aeruginosa-induced hBD-2 expression in intestinal epithelial cells (Figures 4 and 6). Furthermore, NOD1 expressed by epithelial cells takes part in the activation of NF- $\mathrm{kB}$ and the up-regulated production of an 
important epithelial cell chemoattractant in response to $P$. aeruginosa [44]. Gram-negative bacteria can deliver peptidoglycan to cytosolic NOD1 in host cells via a novel mechanism involving outer membrane vesicles (OMVs) including the Gram-negative mucosal pathogens [45]: Helicobacter pylori, Pseudomonas aeruginosa and Neisseria gonorrhoea. These peptidoglycan-containing OMVs enter epithelial cells through lipid rafts, thereby upregulating NF-kB and inducing NOD1-dependent responses in vitro. Future studies should clarify the role of mucosal NOD1 during $P$. aeruginosa infection in vivo.

\section{Conclusion}

In conclusion, we demonstrated $P$. aeruginosa induced proinflammatory responses and antimicrobial peptide in IECs. Prolonged infection by $P$. aeruginosa results in suppression of IL- 8 but enhancement of hBD-2 protein production in SW480 cells, even though both mRNAs were increased gradually. The PI3K/Akt and ERK signaling pathway may be involved in the negative regulation of $P$. aeruginosa-induced IL-8 production in SW480 cells while NOD1 protein was involved in the positive regulation of $P$. aeruginosa-induced hBD-2 expression.

While antimicrobial peptide in epithelial cells has been shown to continuously protect the host against prolonged infection, modulation of proinflammatory responses protects the host from the detrimental effects of overwhelming inflammation. The dysbiosis between $P$. aeruginosa and other Pseudomonas species or gut microbiota could be directly involved in the pathogenesis of $\mathrm{CD}$. The differential regulation of NOD1 and NOD2 in intestinal mucosa on the commensal and pathogenic bacteria deserves further investigation.

\section{Ethics statement}

This was an entirely in-vitro study that was approved by the Chang Gung University Biosafety Committee.

\section{Additional files}

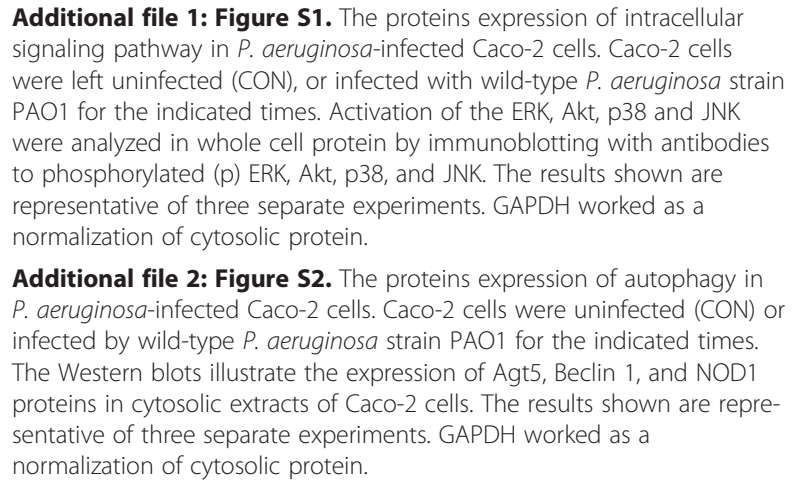

Additional file 2: Figure S2. The proteins expression of autophagy in $P$. aeruginosa-infected Caco-2 cells. Caco-2 cells were uninfected (CON) or infected by wild-type $P$. aeruginosa strain PAO1 for the indicated times. The Western blots illustrate the expression of Agt5, Beclin 1, and NOD1 proteins in cytosolic extracts of Caco-2 cells. The results shown are representative of three separate experiments. GAPDH worked as a normalization of cytosolic protein.

Additional file 3: Figure S3. Involvement of ERK and PI3K/Akt signal pathways in P. aeruginosa-induced IL-8 and hBD-2 in Caco-2 cells. Effect of ERK and PI3K inhibition on P. aeruginosa-induced IL-8 and hBD-2 secretion. Caco-2 cells were left untreated, or treated with 25 MM PD98059 (PD) and $50 \mu \mathrm{M}$ LY294002 (LY) for one hour. They were then infected with the wild-type $P$. aeruginosa strain PAO1. Supernatant was analyzed by ELISA for IL-8 and hBD-2. The amount of IL-8 or hBD-2 produced is shown as the fold increase over control cells (CON). Results are represented as means \pm S.E.M. for at least three determinations from independent experiments. ( ${ }^{*} p<0.05$; \# $p<0.05$ ).

Additional file 4: Figure S4. Involvement of NOD1 in $P$. aeruginosa-induced IL-8 and hBD-2 in Caco-2 cells. Caco-2 cells were transfected with control siRNA and NOD1 siRNA (siRNA = non-target control siRNA; siNod1 = siRNA to NOD1) for 48 hours. The transfected cells were left uninfected or infected by wild-type $P$. aeruginosa strain PAO1. Supernatant was analyzed by ELISA for secreted IL-8 and hBD-2 protein in Caco-2 cells. The amount of IL-8 or hBD-2 produced is shown as the fold increase over control cells (CON). Results are represented as means \pm S.E.M. for at least three determinations from independent experiments. An asterisk indicates a significant difference (* $p<0.05)$.

\section{Competing interests}

The author declares that he has no competing interests.

\section{Author's contributions}

FCH conceived and designed the study, analyzed and interpreted the data, and wrote the manuscript.

\section{Acknowledgements}

The authors wish to thank Dr. You-Lin Tain for his numerous productive discussions. This work was supported in part by National Science Community grant NSC 100-2314-B-182-065 and Chang Gung Medical grant (CMRPG880443 \& CMRPG8B1481).

Received: 17 June 2014 Accepted: 24 October 2014

Published online: 30 November 2014

\section{References}

1. Aliaga L, Mediavilla JD, Cobo F: A clinical index predicting mortality with Pseudomonas aeruginosa bacteraemia. J Med Microbiol 2002, 51(7):615-619.

2. Huang YC, Lin TY, Wang CH: Community-acquired Pseudomonas aeruginosa sepsis in previously healthy infants and children: analysis of forty-three episodes. Pediatr Infect Dis J 2002, 21(11):1049-1052.

3. Kang Cl, Kim SH, Park WB, Lee KD, Kim HB, Kim EC, Oh MD, Choe KW: Clinical features and outcome of patients with community-acquired Pseudomonas aeruginosa bacteraemia. Clin Microbiol Infect 2005, 11(5):415-418

4. Grisaru-Soen G, Lerner-Geva L, Keller N, Berger H, Passwell JH, Barzilai A: Pseudomonas aeruginosa bacteremia in children: analysis of trends in prevalence, antibiotic resistance and prognostic factors. Pediatr Infect Dis J 2000, 19(10):959-963.

5. Strateva T, Yordanov D: Pseudomonas aeruginosa - a phenomenon of bacterial resistance. J Med Microbiol 2009, 58(Pt 9):1133-1148.

6. Stadnyk AW: Intestinal epithelial cells as a source of inflammatory cytokines and chemokines. Can J Gastroenterol 2002, 16(4):241-246.

7. Hecht G: Innate mechanisms of epithelial host defense: spotlight on intestine. Am J Physiol 1999, 277(3 Pt 1):C351-C358.

8. Fleckenstein JM, Kopecko DJ: Breaching the mucosal barrier by stealth: an emerging pathogenic mechanism for enteroadherent bacterial pathogens. J Clin Invest 2001, 107(1):27-30.

9. Akira S, Uematsu S, Takeuchi O: Pathogen recognition and innate immunity. Cell 2006, 124(4):783-801.

10. Ting JP, Davis BK: CATERPILLER: a novel gene family important in immunity, cell death, and diseases. Annu Rev Immunol 2005, 23:387-414.

11. Cigana C, Lore NI, Bernardini ML, Bragonzi A: Dampening host sensing and avoiding recognition in pseudomonas aeruginosa pneumonia. J Biomed Biotechnol 2011, 2011:852513.

12. Gerstel U, Czapp M, Bartels J, Schroder JM: Rhamnolipid-induced shedding of flagellin from Pseudomonas aeruginosa provokes hBD-2 and IL-8 response in human keratinocytes. Cell Microbiol 2009, 11(5):842-853. 
13. Abreu MT, Fukata M, Arditi M: TLR signaling in the gut in health and disease. J Immunol 2005, 174(8):4453-4460.

14. Kim JG, Lee SJ, Kagnoff MF: Nod1 is an essential signal transducer in intestinal epithelial cells infected with bacteria that avoid recognition by toll-like receptors. Infect Immun 2004, 72(3):1487-1495.

15. Travassos LH, Carneiro LA, Ramjeet M, Hussey S, Kim YG, Magalhaes JG, Yuan L, Soares F, Chea E, Le Bourhis L, Boneca IG, Allaoui A, Jones NL, Nunez G, Girardin SE, Philpott DJ: Nod1 and Nod2 direct autophagy by recruiting ATG16L1 to the plasma membrane at the site of bacterial entry. Nat Immunol 2010, 11(1):55-62.

16. Cooney R, Baker J, Brain O, Danis B, Pichulik T, Allan P, Ferguson DJ, Campbell BJ, Jewell D, Simmons A: NOD2 stimulation induces autophagy in dendritic cells influencing bacterial handling and antigen presentation. Nat Med 2010, 16(1):90-97.

17. Boughan PK, Argent RH, Body-Malapel M, Park JH, Ewings KE, Bowie AG, Ong SJ, Cook SJ, Sorensen OE, Manzo BA, Inohara N, Klein NJ, Nunez G, Atherton JC, Bajaj-Elliott M: Nucleotide-binding oligomerization domain-1 and epidermal growth factor receptor: critical regulators of beta-defensins during Helicobacter pylori infection. J Biol Chem 2006, 281(17):11637-11648.

18. Girardin SE, Tournebize R, Mavris M, Page AL, Li X, Stark GR, Bertin J, DiStefano PS, Yaniv M, Sansonetti PJ, Philpott DJ: CARD4/Nod1 mediates NF-kappaB and JNK activation by invasive Shigella flexneri. EMBO Rep 2001, 2(8):736-742

19. Venza I, Visalli M, Cucinotta M, Teti D, Venza M: NOD2 triggers PGE2 synthesis leading to IL-8 activation in Staphylococcus aureus-infected human conjunctival epithelial cells. Biochem Biophys Res Commun 2013, 440(4):551-557.

20. Huang FC: Regulation of Salmonella flagellin-induced interleukin-8 in intestinal epithelial cells by muramyl dipeptide. Cell Immunol 2012, 278(1-2):1-9.

21. Huang FC: Plasma membrane cholesterol plays a critical role in the Salmonella-induced anti-inflammatory response in intestinal epithelial cells. Cell Immunol 2011, 271(2):480-487.

22. Huang FC: Upregulation of Salmonella-induced IL-6 production in Caco-2 cells by PJ-34, PARP-1 inhibitor: involvement of PI3K, p38 MAPK, ERK JNK, and NF-kappaB. Mediators Inflamm 2009, 2009:103890.

23. Huang FC, Werne A, Li Q, Galyov EE, Walker WA, Cherayil BJ: Cooperative interactions between flagellin and SopE2 in the epithelial interleukin-8 response to Salmonella enterica serovar typhimurium infection. Infect Immun 2004, 72(9):5052-5062.

24. Huang FC, Li Q, Cherayil BJ: A phosphatidyl-inositol-3-kinase-dependent anti-inflammatory pathway activated by Salmonella in epithelial cells. FEMS Microbiol Lett 2005, 243(1):265-270.

25. Tsai WC, Strieter RM, Mehrad B, Newstead MW, Zeng X, Standiford TJ: CXC chemokine receptor CXCR2 is essential for protective innate host response in murine Pseudomonas aeruginosa pneumonia. Infect Immun 2000, 68(7):4289-4296.

26. Elewaut D, DiDonato JA, Kim JM, Truong F, Eckmann L, Kagnoff MF: NF-kappa $B$ is a central regulator of the intestinal epithelial cell innate immune response induced by infection with enteroinvasive bacteria. J Immunol 1999, 163(3):1457-1466

27. Joseph T, Look D, Ferkol T: NF-kappaB activation and sustained IL-8 gene expression in primary cultures of cystic fibrosis airway epithelial cells stimulated with Pseudomonas aeruginosa. Am J Physiol Lung Cell Mol Physiol 2005, 288(3):L471-L479.

28. Hazlett LD: Corneal response to Pseudomonas aeruginosa infection. Prog Retin Eye Res 2004, 23(1):1-30.

29. Venza I, Cucinotta M, Visalli M, De Grazia G, Oliva S, Teti D: Pseudomonas aeruginosa induces interleukin-8 (IL-8) gene expression in human conjunctiva through the recruitment of both RelA and CCAAT/enhancer-binding protein beta to the IL-8 promoter. J Biol Chem 2009, 284(7):4191-4199.

30. Oka M, Norose K, Matsushima K, Nishigori C, Herlyn M: Overexpression of IL-8 in the cornea induces ulcer formation in the SCID mouse. $\mathrm{Br}\rfloor$ Ophthalmol 2006, 90(5):612-615.

31. Kierbel A, Gassama-Diagne A, Mostov K, Engel JN: The phosphoinositol-3kinase-protein kinase B/Akt pathway is critical for Pseudomonas aeruginosa strain PAK internalization. Mol Biol Cell 2005, 16(5):2577-2585.

32. Guha M, Mackman N: The phosphatidylinositol 3-kinase-Akt pathway limits lipopolysaccharide activation of signaling pathways and expression of inflammatory mediators in human monocytic cells. $J$ Biol Chem 2002, 277(35):32124-32132

33. Sun $M$, Zhu M, Chen K, Nie X, Deng Q, Hazlett LD, Wu Y, Li M, Wu M, Huang $X$ : TREM-2 promotes host resistance against Pseudomonas aeruginosa infection by suppressing corneal inflammation via a PI3K/Akt signaling pathway. Invest Ophthalmol Vis Sci 2013, 54(5):3451-3462.

34. Williams DL, Li C, Ha T, Ozment-Skelton T, Kalbfleisch JH, Preiszner J, Brooks L, Breuel K, Schweitzer JB: Modulation of the phosphoinositide 3-kinase pathway alters innate resistance to polymicrobial sepsis. J Immunol 2004, 172(1):449-456.

35. Lapaquette $P$, Darfeuille-Michaud A: Abnormalities in the handling of intracellular bacteria in Crohn's disease. J Clin Gastroenterol 2010, 44(Suppl 1):S26-S29.

36. Aldhous MC, Noble CL, Satsangi J: Dysregulation of human beta-defensin-2 protein in inflammatory bowel disease. PLoS One 2009, 4(7):e6285.

37. Zilbauer M, Jenke A, Wenzel G, Postberg J, Heusch A, Phillips AD, Noble-Jamieson G, Torrente F, Salvestrini C, Heuschkel R, Wirth S: Expression of human beta-defensins in children with chronic inflammatory bowel disease. PLoS One 2010, 5(10):e15389.

38. Wagner J, Short K, Catto-Smith AG, Cameron DJ, Bishop RF, Kirkwood CD: Identification and characterisation of Pseudomonas 165 ribosomal DNA from ileal biopsies of children with Crohn's disease. PLoS One 2008, 3(10):e3578.

39. Vasseur F, Sendid B, Jouault T, Standaert-Vitse A, Dubuquoy L, Francois N, Gower-Rousseau C, Desreumaux P, Broly F, Vermeire S, Colombel JF, Poulain D: Variants of NOD1 and NOD2 genes display opposite associations with familial risk of Crohn's disease and anti-saccharomyces cerevisiae antibody levels. Inflamm Bowel Dis 2012, 18(3):430-438.

40. Van Limbergen J, Nimmo ER, Russell RK, Drummond HE, Smith L, Anderson $\mathrm{NH}$, Davies G, Arnott ID, Wilson DC, Satsangi J: Investigation of NOD1/CARD4 variation in inflammatory bowel disease using a haplotype-tagging strategy. Hum Mol Genet 2007, 16(18):2175-2186.

41. Cigana C, Curcuru L, Leone MR, lerano T, Lore NI, Bianconi I, Silipo A Cozzolino F, Lanzetta R, Molinaro A, Bernardini ML, Bragonzi A: Pseudomonas aeruginosa exploits lipid $A$ and muropeptides modification as a strategy to lower innate immunity during cystic fibrosis lung infection. PLoS One 2009, 4(12):e8439.

42. Scharf S, Hippenstiel S, Flieger A, Suttorp N, N'Guessan PD: Induction of human beta-defensin-2 in pulmonary epithelial cells by Legionella pneumophila: involvement of TLR2 and TLR5, p38 MAPK, JNK, NF-kappaB, and AP-1. Am J Physiol Lung Cell Mol Physiol 2010, 298(5):L687-L695.

43. Krisanaprakornkit S, Kimball JR, Dale BA: Regulation of human beta-defensin-2 in gingival epithelial cells: the involvement of mitogen-activated protein kinase pathways, but not the NF-kappaB transcription factor family. $\mathrm{J}$ Immunol 2002, 168(1):316-324.

44. Travassos LH, Carneiro LA, Girardin SE, Boneca IG, Lemos R, Bozza MT, Domingues RC, Coyle AJ, Bertin J, Philpott DJ, Plotkowski MC: Nod1 participates in the innate immune response to Pseudomonas aeruginosa. J Biol Chem 2005, 280(44):36714-36718.

45. Kaparakis M, Turnbull L, Carneiro L, Firth S, Coleman HA, Parkington HC, Le Bourhis L, Karrar A, Viala J, Mak J, Hutton ML, Davies JK, Crack PJ, Hertzog PJ, Philpott DJ, Girardin SE, Whitchurch CB, Ferrero RL: Bacterial membrane vesicles deliver peptidoglycan to NOD1 in epithelial cells. Cell Microbiol 2010, 12(3):372-385.

\section{doi:10.1186/s12866-014-0275-6}

Cite this article as: Huang: Differential regulation of interleukin- 8 and human beta-defensin 2 in Pseudomonas aeruginosa-infected intestinal epithelial cells. BMC Microbiology 2014 14:275. 